\title{
Common T-Cell-Receptor Motifs and Features in Patients with Cytomegalovirus (CMV)-Seronegative End-Stage Renal Disease Receiving a Peptide Vaccination against CMV
}

\author{
Lukas Bunse ${ }^{1,2,+} \mathbb{D}$, Claudia Sommerer ${ }^{3,+}$, Chin Leng Tan ${ }^{1,4}$, Felix Korell ${ }^{5} \mathbb{D}$, Anita Schmitt ${ }^{5}$, \\ Angela Hückelhoven-Krauss ${ }^{5}$, Brigitte Neuber ${ }^{5}$, Thomas Mertens ${ }^{6}$, Michael Platten 1,2,7 (D), Edward W. Green ${ }^{1}$, \\ Martin Zeier ${ }^{3}$ and Michael Schmitt ${ }^{5, *(D)}$
}

Citation: Bunse, L.; Sommerer, C.; Tan, C.L.; Korell, F.; Schmitt, A

Hückelhoven-Krauss, A.; Neuber, B.; Mertens, T.; Platten, M.; Green, E.W.; et al. Common T-Cell-Receptor Motifs and Features in Patients with Cytomegalovirus (CMV)-Seronegative End-Stage Renal Disease Receiving a Peptide Vaccination against CMV. Int J. Mol. Sci. 2022, 23, 1029. https:// doi.org/10.3390/ijms23031029

Academic Editor: Vera Rebmann

Received: 1 December 202

Accepted: 14 January 2022

Published: 18 January 2022

Publisher's Note: MDPI stays neutral with regard to jurisdictional claims in published maps and institutional affiliations.

Copyright: (C) 2022 by the authors. Licensee MDPI, Basel, Switzerland. This article is an open access article distributed under the terms and conditions of the Creative Commons Attribution (CC BY) license (https:// creativecommons.org/licenses/by/ $4.0 /)$
1 DKTK CCU Neuroimmunology and Brain Tumor Immunology, German Cancer Research Center (DKFZ), 69120 Heidelberg, Germany; lukas.bunse@umm.de (L.B.); c.tan@dkfz-heidelberg.de (C.L.T.); michael.platten@umm.de (M.P.); e.green@dkfz-heidelberg.de (E.W.G.)

2 Department of Neurology, MCTN, Medical Faculty Mannheim, Heidelberg University, 69117 Heidelberg, Germany

3 Division of Nephrology, University Hospital Heidelberg, Renal Clinic Heidelberg, 69120 Heidelberg, Germany; claudia_sommerer@web.de (C.S.); Martin.Zeier@med.uni-heidelberg.de (M.Z.)

4 Faculty of Biosciences, Heidelberg University, 69117 Heidelberg, Germany

5 Department of Internal Medicine V, University Hospital Heidelberg, 69120 Heidelberg, Germany; Felix.Korell@med.uni-heidelberg.de (F.K.); Anita.Schmitt@med.uni-heidelberg.de (A.S.); Angela.Hueckelhoven-Krauss@med.uni-heidelberg.de (A.H.-K.); Brigitte.Neuber@med.uni-heidelberg.de (B.N.)

6 Institute of Virology, University of Ulm, 89081 Ulm, Germany; thomas.mertens@uni-ulm.de

7 Immune Monitoring Unit, DKFZ and National Center for Tumor Diseases, 69120 Heidelberg, Germany

* Correspondence: michael.schmitt@med.uni-heidelberg.de; Tel.: +49-6221-56-6614; Fax: +49-6221-56-5740

$+\quad$ These authors contributed equally.

Abstract: After solid-organ transplantation, reactivation of the cytomegalovirus (CMV) is often observed in seronegative patients and associated with a high risk of disease and mortality. CMVspecific $T$ cells can prevent CMV reactivation. In a phase 1 trial, CMV-seronegative patients with end-stage renal disease listed for kidney transplantation were subjected to CMV phosphoprotein 65 (CMVpp65) peptide vaccination and further investigated for T-cell responses. To this end, CMVspecific $\mathrm{CD}^{+} \mathrm{T}$ cells were characterized by bulk T-cell-receptor (TCR) repertoire sequencing and combined single-cell RNA and TCR sequencing. In patients mounting an immune response to the vaccine, a common SYE(N)E TCR motif known to bind CMVpp65 was detected. CMV-peptidevaccination-responder patients had TCR features distinct from those of non-responders. In a nonresponder patient, a monoclonal inflammatory T-cell response was detected upon CMV reactivation. The identification of vaccine-induced CMV-reactive TCRs motifs might facilitate the development of cellular therapies for patients wait-listed for kidney transplantation.

Keywords: CMV; end-stage renal disease; TCR motif; peptide vaccination; single-cell sequencing

\section{Introduction}

Patients receiving a kidney transplantation are prone to cytomegalovirus (CMV) reactivation. The most vulnerable time period is early after transplantation when patients are under high immunosuppression [1], where up to half of these patients can develop symptomatic CMV disease. CMV reactivation and disease are associated with increased long-term morbidity, graft loss $[2,3]$ and mortality $[2,3]$. Following transplantation, patients with the serostatus donor ${ }^{+}$recipient ${ }^{-}\left(\mathrm{D}^{+} \mathrm{R}^{-}\right)$are at highest risk, while $\mathrm{D}^{+} \mathrm{R}^{+}$and $\mathrm{D}^{-} \mathrm{R}^{+}$ transplantations constitute an intermediate risk.

Classical prophylaxis or preemptive therapy using (val)ganciclovir or foscavir is effective, with nephron- and myelotoxicity limiting the use of these drugs [4]. 
In addition to drug treatment, vaccination offers an intriguing option in development for patients at risk [5]: several CMV vaccines are currently under investigation in phase I to phase III clinical trials, featuring attenuated viruses and truncated proteins as well as DNA vaccines [6-11]. The cellular immune response is essential for controlling CMV infection [12]. Patients might be protected once a detectable T-cell response against CMV has been reached.

Recently, we published the results of a first phase I trial for a CMVpp65-derived vaccine in HLA-A*02-positive $\mathrm{CMV}$-seronegative end-stage renal disease patients on the kidney transplant waiting list [13]. The study demonstrated that the HLA-A*02-restricted CMVpp65-peptide vaccine application was safe, was well tolerated and showed clinically encouraging results in these high-risk patients.

However, further investigation of both the affected patient cohort and of possible response factors is highly warranted.

For this reason, we aimed at a deeper understanding of T-cell-receptor (TCR) repertoire dynamics and specificities and, in addition, report here on the characterization of CMVspecific CD8 ${ }^{+} \mathrm{T}$ cells by bulk TCR sequencing as well as combined single-cell RNA and TCR sequencing.

\section{Results}

\subsection{Impact of CMV-Peptide Vaccination on the TCR Repertoire}

To understand the clonal evolution of T-cell clonotypes following repetitive CMVpp65 peptide vaccinations and to identify TCR sequences dominating peptide-induced peripheral T-cell responses, we performed deep repertoire TCRA and TCRB sequencing. In both responder and non-responder patients, repetitive vaccinations were not associated with consistent longitudinal alterations in the most-abundant clonotypes in the peripheral blood (Figure 1). One responder patient (\#007) had a number of dominant clones in the pre-vaccination repertoire, suggesting a pre-existing immune response to an independent immune challenge, which decreased over time. In contrast, the top clonotypes in non-responder patients \#001 and \#002 were stable during repetitive vaccination. Taken together, in our study, the repetitive CMVpp65 peptide vaccination of patients with CMVseronegative end-stage renal disease did not alter the frequency of the most-abundant TCR clonotypes in the peripheral blood. 


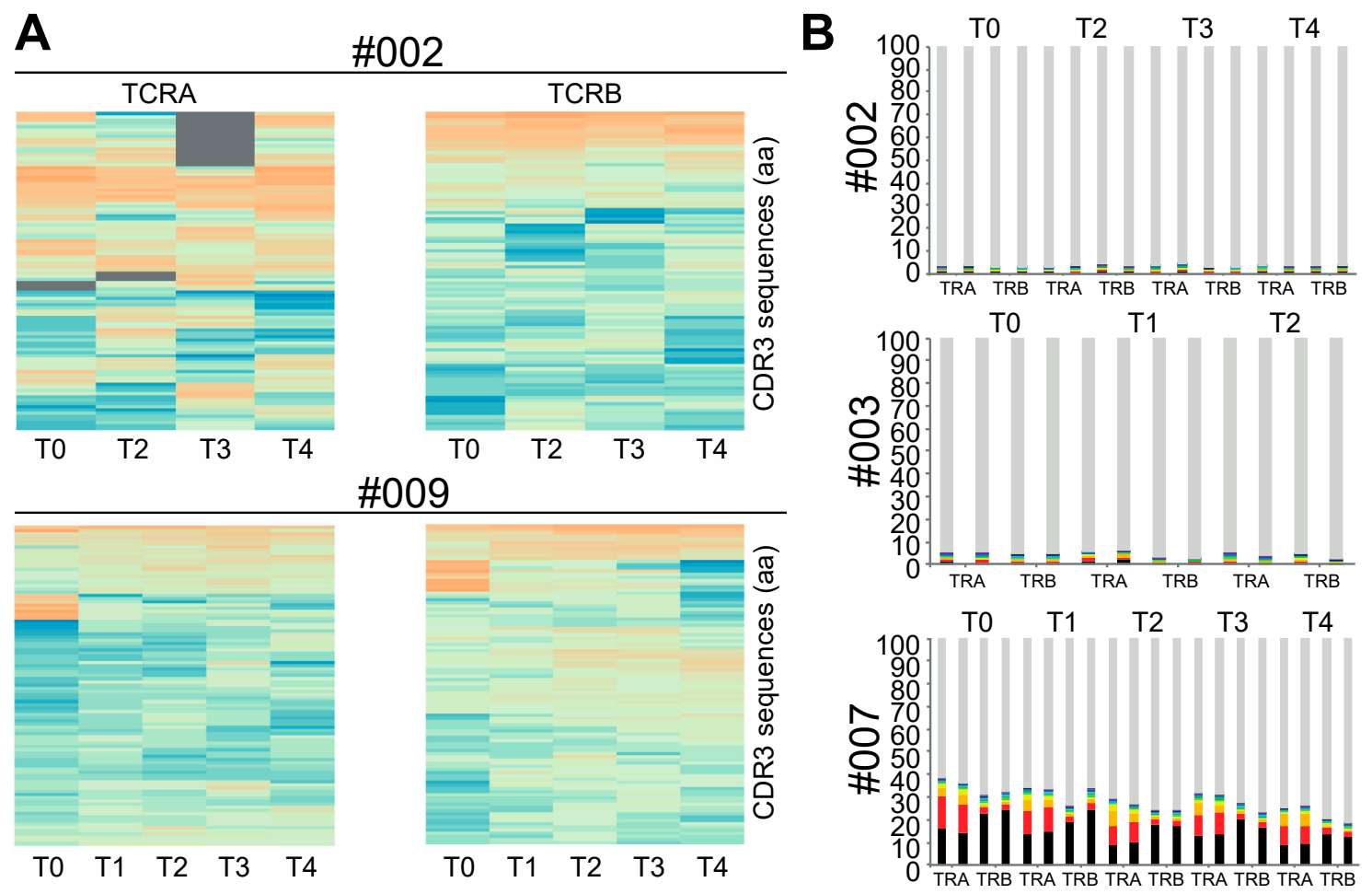

Figure 1. Deep TCRA and TCRB sequencing for patients' PBMCs in CMV-seronegative end-stage renal disease. (A) Top 100 TCRA and TCRB sequences from responder (\#009) and non-responder (\#002) patients visualized at baseline (T0) and three post-vaccine timepoints (T2, T3 and T4). Two exemplary patients are shown. Gray, respective clonotypes were not found at time points illustrated. (B) Proportion of the top-ten clonotypes from responder (\#003, \#007) and non-responder (\#002) patients visualized at baseline (T0) and two to four different post-vaccine timepoints (T1-4). Samples were sequenced in technical duplicates.

To assess the impact of CMVpp65 peptide vaccination on the TCR repertoire in more detail, we performed unsupervised deep-learning-based clustering of patient TCR repertoires using DeepTCR [14]. The featurization of TCRs enables the identification of antigenspecific TCR features in biologically noisy TCR repertoires with a high abundance of irrelevant T cells. Additionally, pre-vaccination TCR features can then be interrogated as predictors of response. Whereas patients \#007 and \#001 each had unique enrichments of CDR3 features throughout the TCR repertoire distinct from all the other patients receiving CMVpp65 peptide vaccination, the remaining eight patients globally showed similar CDR3 feature enrichment within their repertoires including the pre-vaccination time point samples (T0). However, the retrospective classification of response (\#003, \#005, \#006 and $\# 009)$ and non-response (\#002, \#004, \#008 and \#010) by CMV-tetramer staining enabled the identification of distinct response-associated CDR3 features (Figure 2). More specifically, a "SYE(N)E" TCR motif known to bind HLA-A*02-presented pp65 (vdjdb.cdr3.net (accessed on 15 August 2019) was found in responders, making it a predictor of response (Figure 3). 


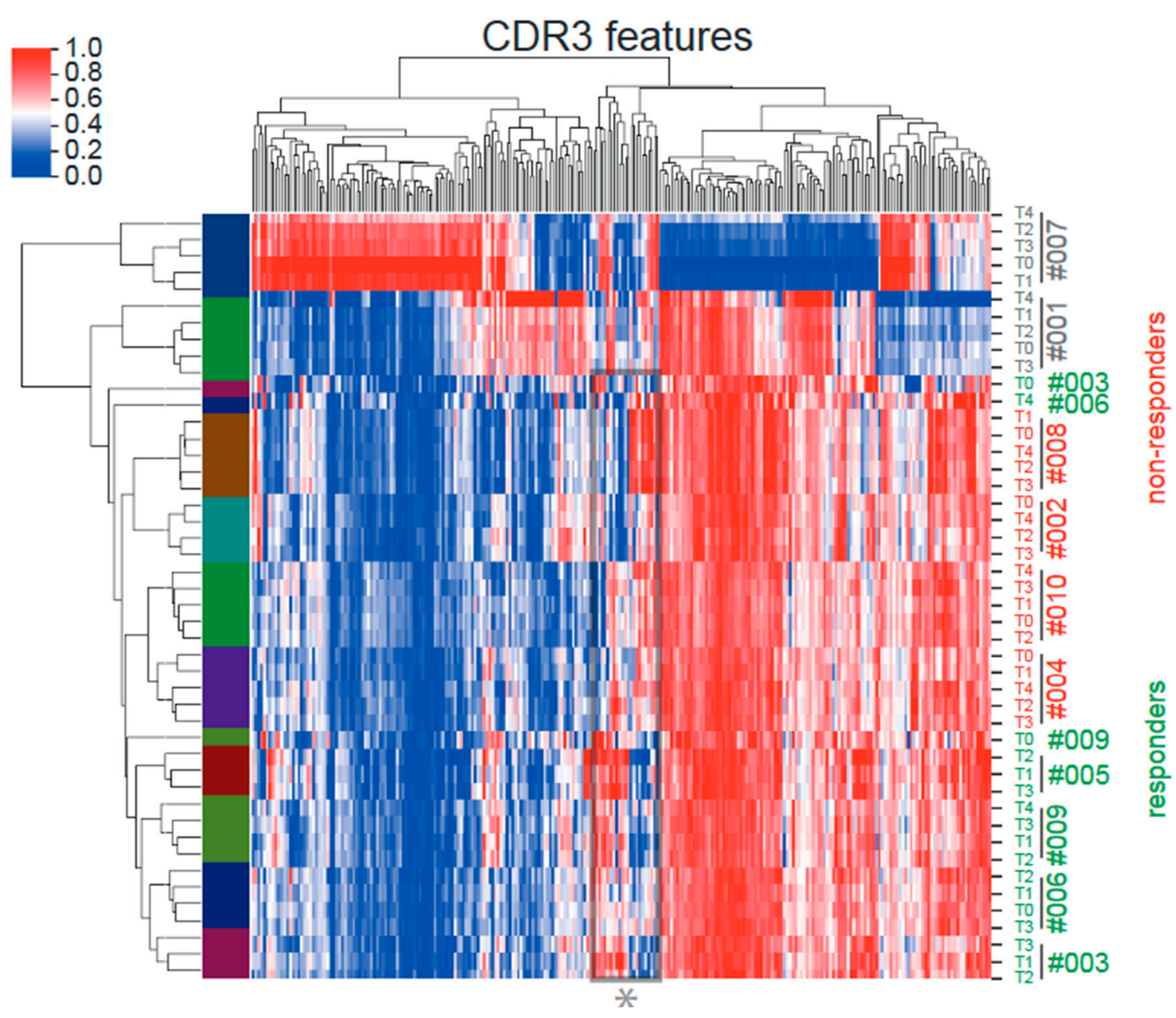

Figure 2. Unsupervised deep-learning-based clustering of patient repertoires using DeepTCR. Ten CMV-seronegative end-stage renal disease patients waiting for kidney transplantation were vaccinated four times biweekly. \#001 and \#007 represent patients with existing strong immune responses. Patients classified as responders (\#003, \#005, \#006 and \#009; green labels) and non-responders (\#002, $\# 004, \# 008$ and \#010; red labels) by CMV-tetramer binding formed distinct clades, with a number of CDR3 features $\left(^{*}\right)$ best correlating with response.
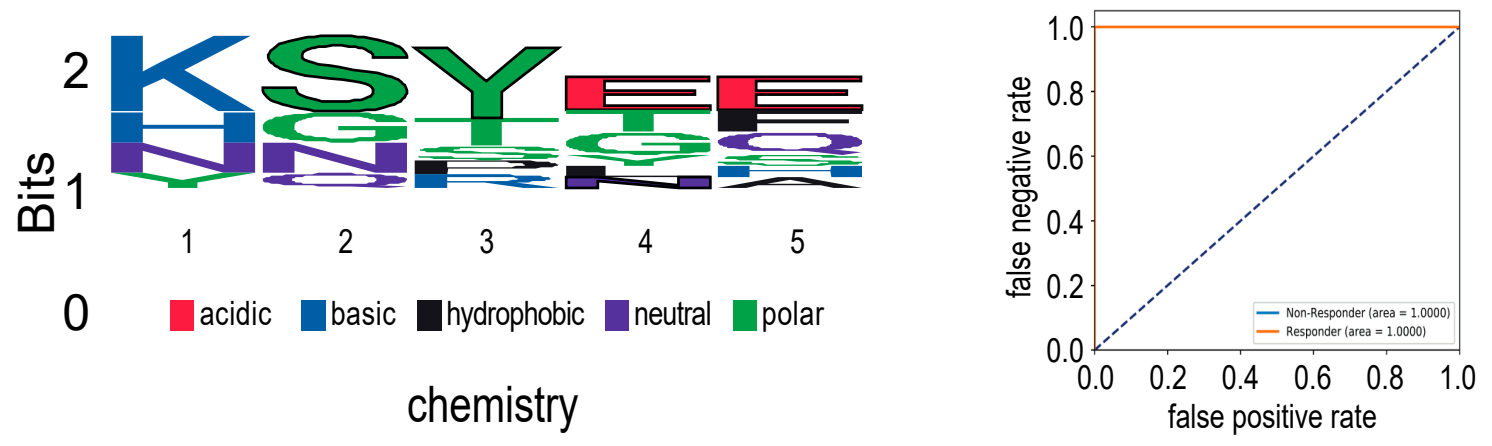

Figure 3. A "SYE(N)E" motif (left) harboring polar, acidic and neutral amino acids known to bind HLA-A*02-presented CMVpp65 was found only in responders but not in non-responder patients (right). 


\subsection{Combined Single RNA and TCR Sequencing in Follow-Up}

Five months after transplantation, non-responder patient \#002 experienced CMV reactivation. To obtain a more detailed immunological understanding of this reactivation, we performed combined single-cell (sc) RNA (scRNA) and scTCR sequencing of CMV-reactive PBMC-derived T cells. Upon reactivation, the patient mounted a monoclonal (80\%) CMV-specific T-cell response with a unique TCR beta chain pairing with two different alpha chains (Figure 4). Furthermore, the combined scRNA/scTCR-seq of the patient \#002-dominating $\mathrm{CD}^{+} \mathrm{CMV}$-specific clonotype revealed two predominant transcriptional states highlighted by a high degree of both effector function (NKG7 $7^{\text {high }}$, CX3CR $1^{\text {high }}$ and GZMB ${ }^{\text {high }}$ ) $[15,16]$ and proliferative capacity (TYMS ${ }^{\text {high }}$ and MKI67 high) (Figure 5, Supplementary Table S1). Using the software package ALICE [17], calculating the generation probability for CDR3s using OLGA [18], we further explored whether other signals of convergent CDR3 selection in patient \#002 occurred during repetitive CMVpp65 and CMV reactivation. Interestingly, we found evidence of multiple convergently selected CDR3 sequences such as CDR3, CASSAGTGTYEQY, which was previously reported to bind the IE1 'KLGGALQAK' epitope presented on HLA-A*03 (vdjdb.cdr3.net (accessed on 1 November 2021)).
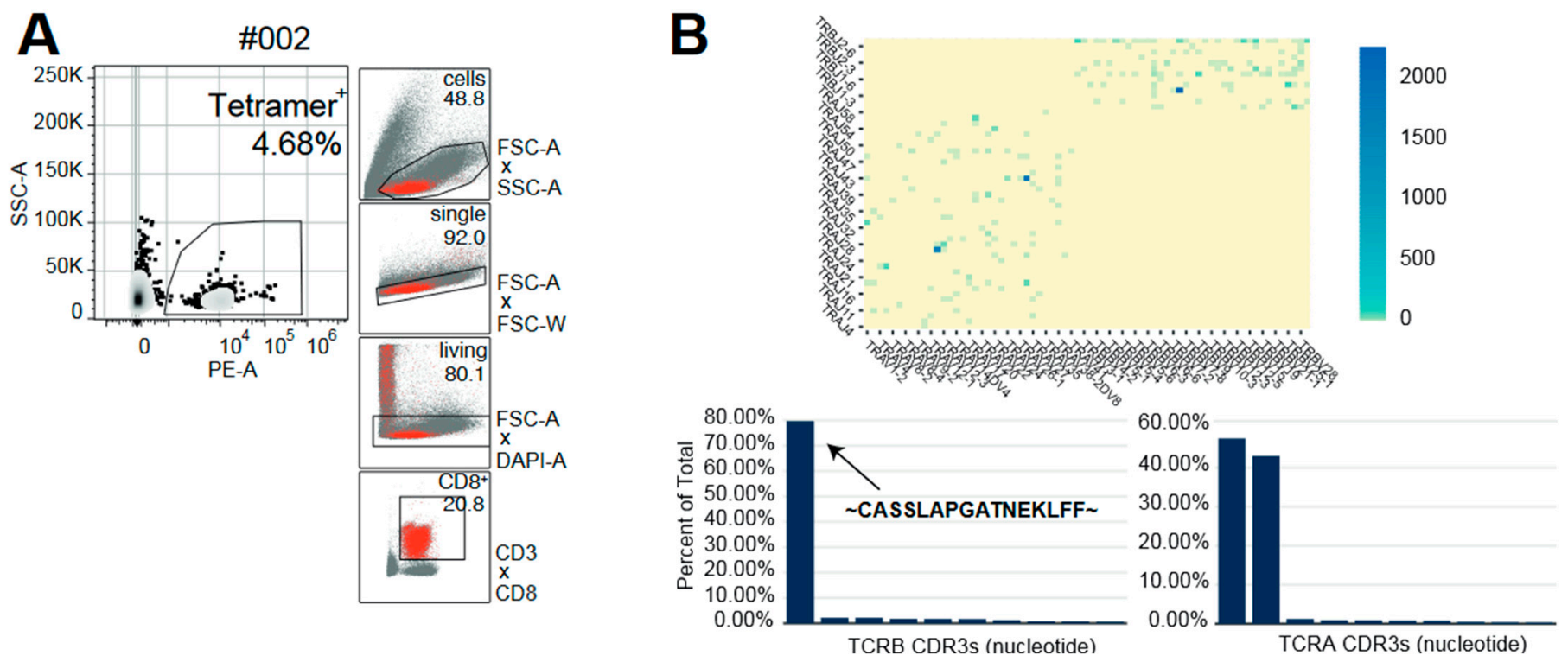

Figure 4. Single-cell TCR sequencing upon CMV reactivation in non-responder patient \#002 five months after transplantation. Combined scTCR-seq and scRNA-seq for HLA-A2*-tetramer-sorted CMV-reactive circulating peripheral $\mathrm{T}$ cells after CMV reactivation. (A) Tetramer sorting gating strategy for CMV-reactive circulating peripheral T cells. (B) TCRA and TCRB gene usage heatmap (left) and clonotype percentages (right) of CMV-reactive T-cell clonotypes. Most-abundant TCR beta chain is illustrated as amino-acid sequence.

In contrast, the scTCR-sequencing of tetramer-sorted CMV-reactive T cells from vaccine-responding patient \#003 revealed that the second-most-abundant post-enrichment clonotype was found at all the available post-vaccine time points, and its abundance peaked, in congruence with the peripheral total T-cell response, at time point 3 (Figure 6). Of note, the most-abundant scTCR-sequencing clonotype from patient \#003 constituting up to $5 \%$ of the tetramer-sorted CMV-reactive TCR repertoire was not found in the deep TCRB PBMC sequencing datasets. 
A

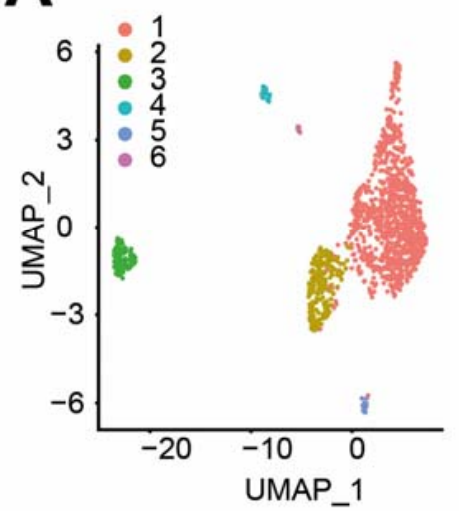

CD3E

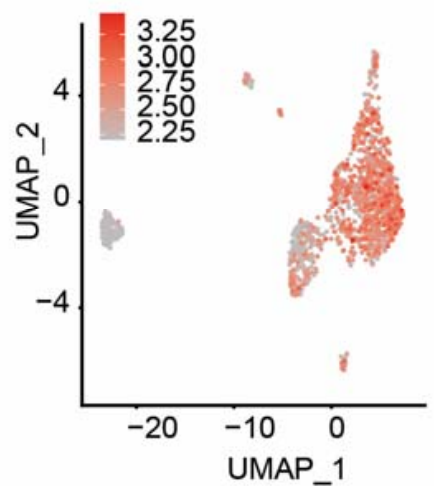

CD8A

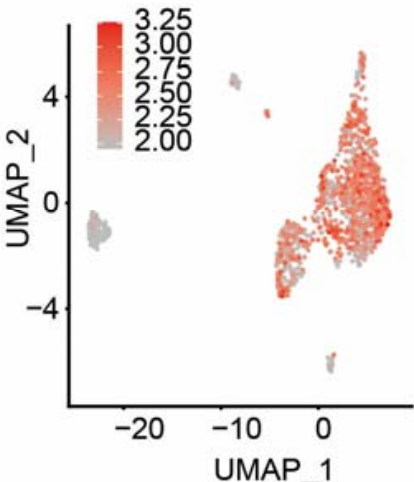

top clonotype (VDJ)

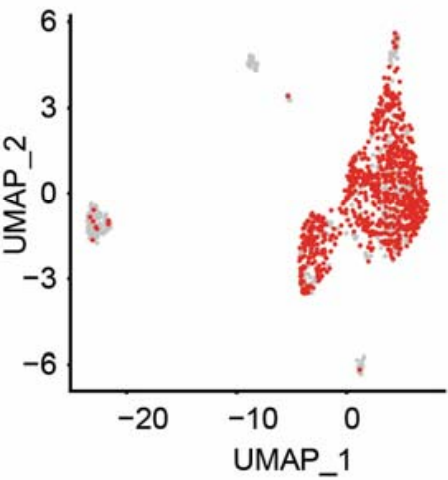

B

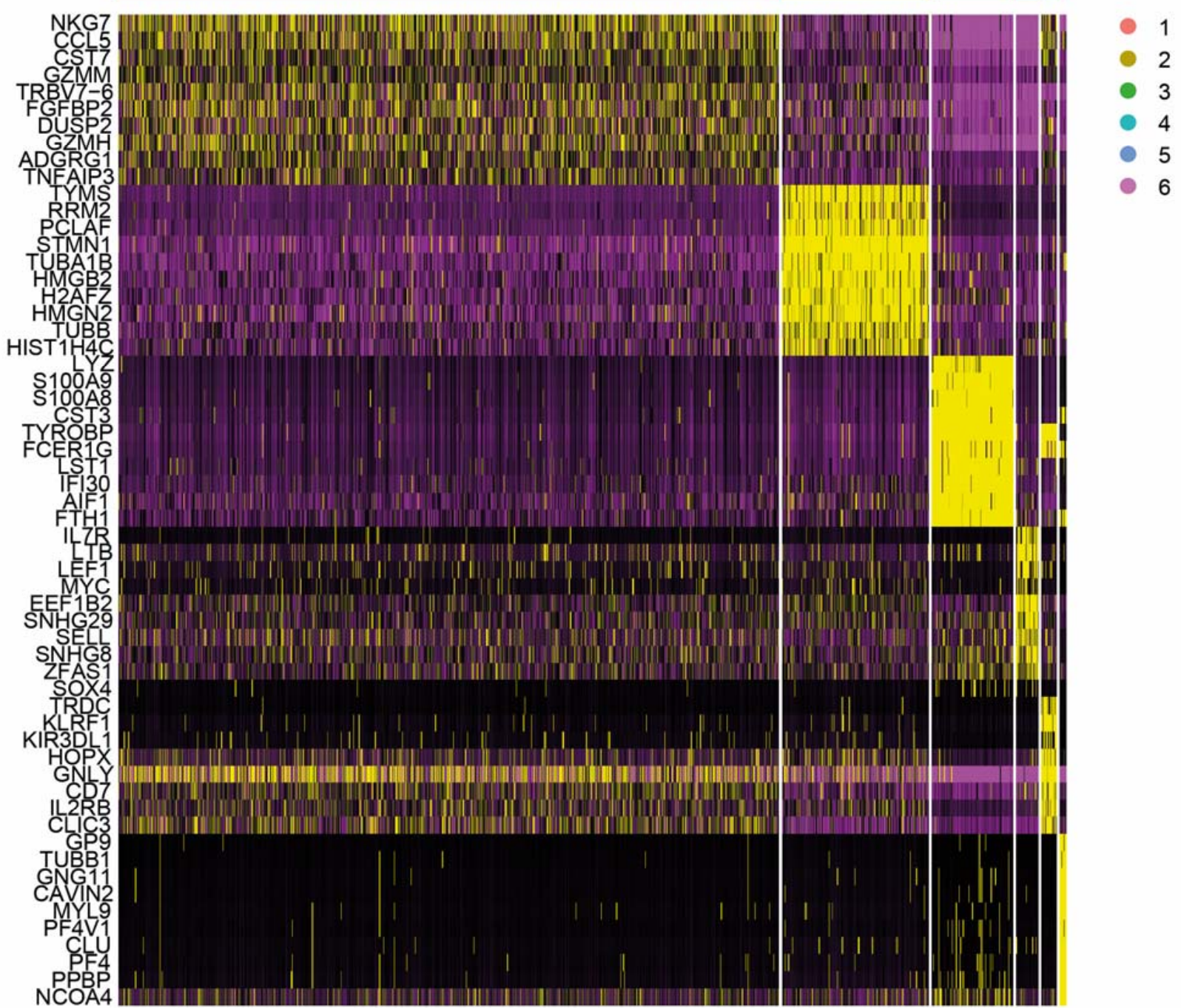

Figure 5. Combined scRNA-seq and scTCR-seq of patient \#002-dominating CD8 ${ }^{+} \mathrm{CMV}$-reactive T-cell clonotype. (A) Six differential T-cell clusters of patient \#002-dominating CD8 ${ }^{+} \mathrm{CMV}$-reactive T-cell clonotypes visualized by UMAP. Expression levels of CD3E and CD8A are shown. Co-visualization of the top TCR clonotype and its distribution within T-cell clusters visualized by UMAP. (B) Heat map of differentially expressed genes in clusters. 

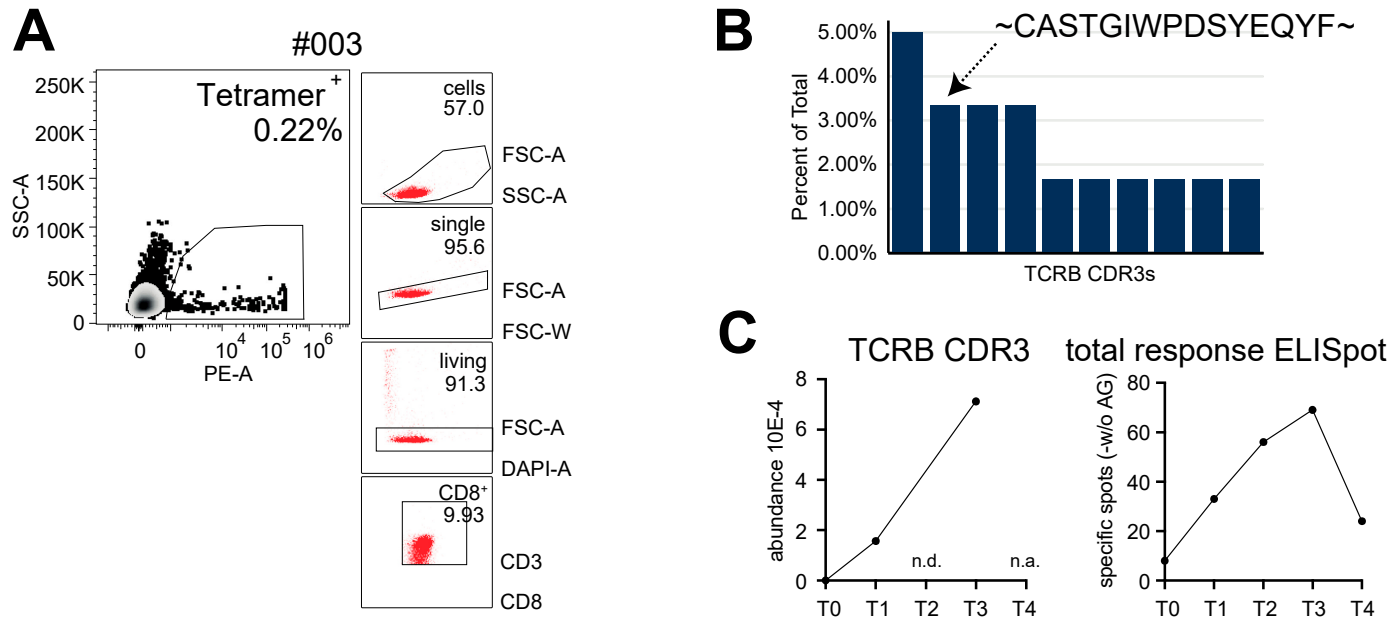

Figure 6. Combined scRNA-seq and scTCR-seq of CMV-reactive T cells from vaccine-responding patient \#003. (A) Tetramer sorting gate for CMV-reactive circulating peripheral T cells. (B) Frequency of top-ten TCR clonotypes within the enriched CMV-reactive T-cell repertoire. Amino-acid sequence of the second-most-abundant clonotype is shown. (C) Clonotypic evolution of the second-mostabundant sc-sequencing-retrieved TCR for longitudinal TCRB deep-sequencing datasets (left) and longitudinal peripheral pp65-specific ELISpot responses (right). n.d., not detectable; n.a., not assessed.

\section{Discussion}

CMV reactivation after solid-organ transplantation constitutes a serious clinical problem [19]. It is known that cellular immunity through effector cytotoxic and helper T cells plays a critical role in controlling CMV replication after transplantation [20].

Within our phase 1 trial, ten patients received four subcutaneous vaccinations as per protocol. The vaccine was well tolerated, and 5/10 patients mounted a T-cell response with an emulsified CMVpp65 nonamer peptide [13]. Further investigation of both the affected patient cohort and of possible response factors is highly warranted.

For this reason, we analyzed the T-cell response to CMV-specific peptide vaccination in exemplary patients in more detail for a deeper understanding of T-cell receptor (TCR) repertoire dynamics and specificities and the characterization of CMV-specific CD8 ${ }^{+} \mathrm{T}$ cells by bulk TCR sequencing as well as combined single-cell RNA and TCR sequencing.

Previous reports had demonstrated that immunodominant CMV antigens can induce highly diverse changes in T-cell-receptor (TCR) repertoires, which, nevertheless, contain convergently selected motifs and/or public clones indicative of patient response and HLA type [21-23]. In both responder and non-responder patients, repetitive vaccinations were not associated with longitudinal alterations in the most-abundant clonotypes in the peripheral blood (Figure 1). Therefore, we performed an unsupervised deep-learning-based clustering of TCR repertoires using DeepTCR [14]. Including pre-vaccination timepoints (T0), responders and non-responders formed distinct clades in TCR cluster analysis (Figure 2). Moreover, a "SYE(N)E" motif known to bind HLA-A*02-presented pp65 was found in responders as a predictor of response (Figure 3). In patient \#002, at five months after transplantation, upon CMV reactivation, we sorted CMV-reactive circulating peripheral T cells (Figure $4 \mathrm{~A}$ ). A single clone constituted $80 \%$ of the repertoire of HLA-A*02 CMVtetramer-positive T cells (Figure 4B). This clonotype was described in several studies as HLA-A*02 restricted and CMV reactive [23] but was not found in longitudinal TCRA and TCRB datasets for the same patient, suggesting an inability to mount a distinct clonotypic response as a mechanism of CMV reactivation. Whereas conclusions on disease severity from this single observation cannot be drawn, the generation of this monoclonal T-cell response might be a consequence of a profound CMV antigenemia that might represent a stronger immunological stimulus compared to the CMVpp65 peptide vaccine. However, we found evidence of multiple convergently selected CDR3 sequences such as CDR3, CAS- 
SAGTGTYEQY, which was previously reported to bind the IE1 'KLGGALQAK' epitope presented on HLA-A*03 (vdjdb.cdr3.net). As patient \#002 is also HLA-A*31 positive and HLA-A $* 3$ and HLA-A*31 fall into the same HLA supertype family, it remains unknown if this observation is causally related to both the previously reported ineffective T-cell response (tetramer staining) but, at the same time, weak T-Track ${ }^{\text {TM }}$ [24] assay results [13].

In general, transplantation-associated immunosuppression interferes with anti-CMV immune responses [20]. Specifically, it has been shown that T-cell-depleting agents increased the risk for CMV infection due to the direct depletion of functional CMV-specific $\mathrm{T}$ cells. Conversely, it has been reported that a release of proinflammatory cytokines is capable of activating latent CMV infections. Mycophenolic acid (MA) inhibits lymphocyte activation that facilitates CMV infection, especially in high doses [25]. MA treatment decreases both primary and secondary humoral immune responses [26]. In a previous study, MA reduced the seroresponse of kidney transplant recipients to pandemic H1N1 vaccination [27]. Importantly, while T-cell-depleting agents were prohibited in this phase 1 clinical study, the use of MA might have influenced the post-vaccination cellular immune responses.

Vaccination strategies have been highly efficacious for several decades in controlling infectious diseases, highlighted more recently by the COVID-19 pandemic. Vaccines that include whole organisms or large proteins appear to have some adverse side effects attributable to the inclusion of an unnecessary antigenic load [28]. In principle, a high antigenic load might increase the probability of allergenic responses. Peptide vaccination, however, is an attractive alternative strategy that relies on the usage of short peptide fragments to engineer the induction of highly targeted immune responses. On the other hand, and in contrast to mRNA vaccines, peptide vaccines are often weakly immunogenic and require adjuvants. In the recent study, the CMVpp65 peptide was emulsified with incomplete Freund's Adjuvant (Montanide ${ }^{\circledR}$ ) and combined with a local application of the Toll-like receptor (TLR)-7 agonist imiquimod (Aldara ${ }^{\circledR} 5 \%$ cream) [13]. Both adjuvants had been used successfully and safely in other studies [29]. It remains unknown if the use of other adjuvants would have resulted in differential outcomes such as in non-responder patient \#002, who demonstrated, in a follow-up analysis, in principle, an ability to mount a meaningful monoclonal response against CMVpp65 solely upon CMV reactivation.

In summary, $50.0 \%$ of the vaccinated patients mounted a peripheral immune response during prophylactic CMV-specific peptide vaccination prior to kidney transplantation. Although only a small number of patients were enrolled in this phase 1 clinical trial, CMVassociated TCR motifs could be identified. Further follow-up studies with an increased patient number and multi-center assessment are necessary to confirm the clinical as well as exploratory translational results. Future vaccination strategies might also incorporate MHC class II antigens or other immunotherapeutic strategies such as mRNA vaccines or TCR-transgenic cellular therapies.

\section{Materials and Methods}

\subsection{Underlying Clinical Study}

Detailed information for both the CMVpp65 peptide vaccine and the investigated patients has been described earlier in the published clinical results of the phase I peptide vaccination trial [13].

In this phase I study, $10 \mathrm{CMV}$-seronegative end-stage renal disease patients waiting for kidney transplantation were vaccinated four times biweekly. All the enrolled patients were CMV IgM/IgG negative prior to the CMVpp65 peptide vaccination. At baseline, the participants showed neither pre-existing CMV-specific CD8 ${ }^{+} \mathrm{T}$ cells in tetramer-based flow cytometry nor significant $(>10 / 200.000)$ interferon gamma (IFN- $\gamma$ ) spot-forming cells (SFC).

In 5 of 10 patients $(50.0 \%$; \#003, \#005, \#006, \#007 and \#009), any immune responses, as evidenced by the detection of an increase in IFN- $\gamma$ production in the T-Track ${ }^{\mathrm{TM}}$ assay and/or an increase in CMV-specific $\mathrm{CD}^{+} \mathrm{T}$ cells, were observed within the core study of 56 days lasting until 14 days after the last vaccination [13]. Patients \#001, \#002, \#004, $\# 008$ and \#010 were non-responder patients. Four patients developed CMV-specific effector 
$\mathrm{T}$ cells, and one patient developed significant immediate-early (IE)-1- and pp65-specific spot-forming cells in the IFN- $\gamma$ ELISpot assay only (\#007).

Details on the manufacturing process and administration of the vaccine as well as all the clinical results and side effects are provided in the previous publication [13].

\subsection{Tetramer Staining for CMV-Specific CD8+ T Cells}

The frequency of CMV-specific $\mathrm{CD} 8^{+} \mathrm{T}$ cells was determined as described earlier [30]. The acquisition was performed on an LSRII ${ }^{\mathrm{TM}}$ device (BD Biosciences, San Diego, CA, USA), and the analysis was performed using the BD FACSDiva ${ }^{\mathrm{TM}}$ software (BD Bioscience, San Diego, CA, USA).

\subsection{Bulk T-Cell-Receptor Repertoire Sequencing and Analysis}

A total of $5 \times 10^{6}$ viable peripheral blood mononuclear cells (PBMCs) per time point for each patient were frozen in DMSO/freezing medium. Following thawing, RNA extraction was performed and the RNA was concentrated using a SpeedVac ${ }^{\mathrm{TM}}$ (Thermo Scientific $^{\mathrm{TM}}$, Waltham, MA, USA). Where sufficient RNA was available, duplicate random amplification of cDNA ends (RACE) reactions targeting the alpha- and beta-chains of the TCR (TCRA and TCRB) were set up using $250 \mathrm{ng}$ of RNA as the template. Preliminary repertoire analysis was conducted with VDJ tools [31], and the CMV specificity was determined with reference to published CMV-specific TCRs listed in VDJdb (June 2017) [31]. The TCR repertoires were further analyzed using the publicly available tools ALICE/OLGA $[17,18]$ and DeepTCR [14].

\subsection{Single-Cell RNA and T-Cell-Receptor Library Construction and Sequencing}

For one responder (patient \#003) and one non-responder (patient \#008), single-cell RNA-seq and single-cell TCR-seq libraries were prepared using the Single Cell Immune Profiling Solution Kit (10× Genomics, Pleasanton, CA, USA), according to the manufacturer's instructions. For the gene-expression library construction, amplified cDNA was fragmented and end-repaired, double-sided size-selected with SPRIselect beads (Beckman Coulter), PCR-amplified with sample-indexing primers (10× Genomics, Pleasanton, CA, USA) and double-sided size-selected with SPRIselect beads (Beckman Coulter, Brea, CA, USA). For TCR library construction, TCR transcripts were enriched from $2 \mu \mathrm{L}$ of amplified cDNA by PCR. Following TCR enrichment, the PCR product was fragmented and end-repaired, size-selected with SPRIselect beads (Beckman Coulter, Brea, CA, USA), PCR-amplified with sample-indexing primers and size-selected with SPRIselect beads (Beckman Coulter, Brea, CA, USA). The single-cell RNA libraries were sequenced on an Illumina HiSeq $4000^{\mathrm{TM}}$ (San Diego, CA, USA). The single-cell TCR libraries were sequenced on an Illumina NextSeq $550^{\mathrm{TM}}$ paired-end 150 mid-output flow cell to a minimum sequencing depth of 5000 reads per cell.

\subsection{Single-Cell Data Analysis}

The sequencing data were aligned using cellranger (v6.1.2) $(10 \times$ Genomics, Pleasanton, CA, USA). The count matrix was then imported into $\mathrm{R}$ for further downstream analysis. The data were normalized and transformed using the Seurat package sctransform and subsequently clustered via the Louvain algorithm using Seurat. A UMAP and heatmap were also plotted using Seurat (v.4.0.4).

\subsection{IFN- $\gamma$ ELISpot}

Commercially available IFN- $\gamma$ ELISpot T-Track ${ }^{\circledR}$ CMV (Lophius Biosciences GmbH, Regensburg, Germany) assays were used for the assessment of CMVpp65 antigen-specific IFN- $\gamma$ release as previously reported [13]. 


\subsection{Statistical Analyses}

The exploratory results are presented in a descriptive manner, with numbers and percentages.

Supplementary Materials: The following are available online at https://www.mdpi.com/article/10 .3390/ijms23031029/s1.

Author Contributions: L.B. performed experiments, analyzed and interpreted data and wrote parts of the manuscript. C.S. designed and conducted the clinical study, collected and analyzed clinical data and wrote parts of the manuscript. E.W.G. performed TCR analyses. F.K. wrote parts of the manuscript, and edited text and figures. A.S. was the head of production for the vaccines. A.H.-K. was responsible for the quality control (QC) of the vaccines and logistics. C.L.T. performed TCR analyses. B.N., T.M., and M.P. discussed the manuscript. M.Z. supervised the study's conception and the performance of the study. M.S. set up the study concept and design, was the qualified person (QP) for the vaccine preparation and wrote the manuscript. All the authors were involved in the study conduct and review of the study data. All the authors have read and agreed to the published version of the manuscript. All authors have read and agreed to the published version of the manuscript.

Funding: The CMVPepVac Study Scientific Committee designed the study, reviewed the study data and statistical analysis and wrote the report. All the authors had full access to the study data, decided to submit the report for publication, and assume responsibility for the completeness and accuracy of the data and the content and integrity of the report. The study was partly funded by the Else KrönerFresenius Foundation, Germany; the Division of Nephrology and Renal Clinic Heidelberg; and the Department of Internal Medicine V, University Hospital Heidelberg. M.P. received funding from the Baden-Württemberg Stiftung (BWST_ISF2018-046). L.B. was funded by the Else Kröner-Fresenius Foundation (2019_EKMS.49) and Deutsche Forschungsgemeinschaft, SFB 1389-B03. C.S. was funded by the Else Kröner-Fresenius Foundation (2014_A151).

Institutional Review Board Statement: The translational analyses were part of the CMVPepVac study. CMVPepVac was conducted according to the guidelines of the Declaration of Helsinki and approved by the Institutional Ethics Committee (IRB No. AFmo-256/2013; date of approval: 10 October 2013) as well as by the Federal Regulatory Authority, the Paul-Ehrlich-Institute, Langen, Germany (PEI registration No. 1855/02; date of approval: 10 October 2013).

Informed Consent Statement: Informed consent was obtained from all the subjects involved in the study.

Data Availability Statement: The data presented in this study are available on request from the corresponding author. The data are not publicly available due to national law.

Acknowledgments: We thank the study coordinator, Ute von der Emde, for excellent study support and Richard Gabriel for bulk T-cell-receptor repertoire sequencing.

Conflicts of Interest: The following authors of this manuscript have conflicts of interest to disclose: Apogenix: Funding for collaborative research. Hexal: Financial support for research on biosimilars and travel grants. Kite: Financial support for educational activities and conference and travel grants. A Co-PI for clinical trials on CAR-T cells. MSD: Ad board member, and a PI of clinical trials on letermovir. Novartis: Collaborative research grant for CAR-T cells. A Co-PI for clinical trials on CAR-T cells. TolerogenixX: Co-founder and shareholder. Conflict of interest (COI) declaration for AS: TolerogenixX: Co-founder and shareholder. Conflict of interest (COI) declaration for CS: Chiesi: Grant for research on biomarkers independent of the present study provided to the institution. Novartis: PI for clinical trials on immunosuppression. Calliditas Therapeutics: PI for a clinical trial on renal disease. All the other authors state no conflict of interest.

\section{References}

1. De Keyzer, K.; Van Laecke, S.; Peeters, P.; Vanholder, R. Human Cytomegalovirus and Kidney Transplantation: A Clinician's Update. Am. J. Kidney Dis. 2011, 58, 118-126. [CrossRef] [PubMed]

2. Sagedal, S.; Hartmann, A.; Nordal, K.P.; Osnes, K.; Leivestad, T.; Foss, A.; Degré, M.; Fauchald, P.; Rollag, H. Impact of early cytomegalovirus infection and disease on long-term recipient and kidney graft survival. Kidney Int. 2004, 66, 329-337. [CrossRef] [PubMed] 
3. Hodson, E.M.; A Jones, C.; Webster, A.C.; Strippoli, G.F.; Barclay, P.G.; Kable, K.; Vimalachandra, D.; Craig, J.C. Antiviral medications to prevent cytomegalovirus disease and early death in recipients of solid-organ transplants: A systematic review of randomised controlled trials. Lancet 2005, 365, 2105-2115. [CrossRef]

4. Helanterä, I.; Kyllönen, L.; Lautenschlager, I.; Salmela, K.; Koskinen, P. Primary CMV Infections Are Common in Kidney Transplant Recipients After 6 Months Valganciclovir Prophylaxis. Arab. Archaeol. Epigr. 2010, 10, 2026-2032. [CrossRef]

5. Plotkin, S.A.; Boppana, S.B. Vaccination against the human cytomegalovirus. Vaccine 2018, 37, 7437-7442. [CrossRef]

6. Hill, A.B. The immune response to CMV infection and vaccination in mice, monkeys and humans: Recent developments. Curr. Opin. Virol. 2018, 28, 161-166. [CrossRef]

7. Nakamura, R.; La Rosa, C.; Longmate, J.; Drake, J.; Slape, C.; Zhou, Q.; Lampa, M.G.; O’Donnell, M.; Cai, J.-L.; Farol, L.; et al. Viraemia, immunogenicity, and survival outcomes of cytomegalovirus chimeric epitope vaccine supplemented with PF03512676 (CMVPepVax) in allogeneic haemopoietic stem-cell transplantation: Randomised phase 1b trial. Lancet Haematol. 2015, 3, e87-e98. [CrossRef]

8. $\quad$ Bernstein, D.I.; Munoz, F.M.; Callahan, S.T.; Rupp, R.; Wootton, S.H.; Edwards, K.M.; Turley, C.B.; Stanberry, L.R.; Patel, S.M.; Mcneal, M.M.; et al. Safety and efficacy of a cytomegalovirus glycoprotein B (gB) vaccine in adolescent girls: A randomized clinical trial. Vaccine 2015, 34, 313-319. [CrossRef] [PubMed]

9. Pass, R.F.; Zhang, C.; Evans, A.; Simpson, T.; Andrews, W.; Huang, M.-L.; Corey, L.; Hill, J.; Davis, E.; Flanigan, C.; et al. Vaccine Prevention of Maternal Cytomegalovirus Infection. N. Engl. J. Med. 2009, 360, 1191-1199. [CrossRef]

10. Wloch, M.K.; Smith, L.R.; Boutsaboualoy, S.; Reyes, L.; Han, C.; Kehler, J.; Smith, H.D.; Selk, L.; Nakamura, R.; Brown, J.M.; et al. Safety and Immunogenicity of a Bivalent Cytomegalovirus DNA Vaccine in Healthy Adult Subjects. J. Infect. Dis. 2008, 197, 1634-1642. [CrossRef] [PubMed]

11. A Kharfan-Dabaja, M.; Boeckh, M.; Wilck, M.B.; A Langston, A.; Chu, A.H.; Wloch, M.K.; Guterwill, D.F.; Smith, L.R.; Rolland, A.P.; Kenney, R.T. A novel therapeutic cytomegalovirus DNA vaccine in allogeneic haemopoietic stem-cell transplantation: A randomised, double-blind, placebo-controlled, phase 2 trial. Lancet Infect. Dis. 2012, 12, 290-299. [CrossRef]

12. Reusser, P.; Riddell, S.R.; Meyers, J.D.; Greenberg, P.D. Cytotoxic T-lymphocyte response to cytomegalovirus after human allogeneic bone marrow transplantation: Pattern of recovery and correlation with cytomegalovirus infection and disease. Blood 1991, 78, 1373-1380. [CrossRef]

13. Sommerer, C.; Schmitt, A.; Hückelhoven-Krauss, A.; Giese, T.; Bruckner, T.; Wang, L.; Schnitzler, P.; Meuer, S.; Zeier, M.; Schmitt, M. Peptide Vaccination Against Cytomegalovirus Induces Specific T Cell Response in Responses in CMV Seronegative End-Stage Renal Disease Patients. Vaccines 2021, 9, 133. [CrossRef]

14. Sidhom, J.-W.; Larman, H.B.; Pardoll, D.M.; Baras, A.S. DeepTCR: A deep learning framework for revealing structural concepts within TCR Repertoire. bioRxiv 2018, 464107. [CrossRef]

15. Gerlach, C.; Moseman, E.A.; Loughhead, S.M.; Alvarez, D.; Zwijnenburg, A.J.; Waanders, L.; Garg, R.; de la Torre, J.C.; von Andrian, U.H. The Chemokine Receptor CX3CR1 Defines Three Antigen-Experienced CD8 T Cell Subsets with Distinct Roles in Immune Surveillance and Homeostasis. Immunity 2016, 45, 1270-1284. [CrossRef]

16. Ng, S.S.; Rivera, F.D.L.; Yan, J.; Corvino, D.; Das, I.; Zhang, P.; Kuns, R.; Chauhan, S.B.; Hou, J.; Li, X.-Y.; et al. The NK cell granule protein NKG7 regulates cytotoxic granule exocytosis and inflammation. Nat. Immunol. 2020, 21, 1205-1218. [CrossRef]

17. Pogorelyy, M.V.; Minervina, A.A.; Shugay, M.; Chudakov, D.M.; Lebedev, Y.B.; Mora, T.; Walczak, A.M. Detecting T cell receptors involved in immune responses from single repertoire snapshots. PLoS Biol. 2019, 17, e3000314. [CrossRef] [PubMed]

18. Sethna, Z.; Elhanati, Y.; Callan, C.G.; Walczak, A.M.; Mora, T. OLGA: Fast computation of generation probabilities of B- and T-cell receptor amino acid sequences and motifs. Bioinformatics 2019, 35, 2974-2981. [CrossRef]

19. Torre-Cisneros, J.; Aguado, J.; Caston, J.; Almenar, L.; Alonso, A.; Cantisán, S.; Carratala, J.; Cervera, C.; Cordero, E.; Fariñas, M.; et al. Management of cytomegalovirus infection in solid organ transplant recipients: SET/GESITRA-SEIMC/REIPI recommendations. Transplant. Rev. 2016, 30, 119-143. [CrossRef] [PubMed]

20. Lúcia, M.; Crespo, E.; Cruzado, J.M.; Grinyó, J.M.; Bestard, O. Human CMV-specific T-cell responses in kidney transplantation; toward changing current risk-stratification paradigm. Transpl. Int. 2014, 27, 643-656. [CrossRef]

21. Huth, A.; Liang, X.; Krebs, S.; Blum, H.; Moosmann, A. Antigen-Specific TCR Signatures of Cytomegalovirus Infection. J. Immunol. 2018, 202, 979-990. [CrossRef]

22. Chen, G.; Yang, X.; Ko, A.; Sun, X.; Gao, M.; Zhang, Y.; Shi, A.; Mariuzza, R.A.; Weng, N.-P. Sequence and Structural Analyses Reveal Distinct and Highly Diverse Human CD8 + TCR Repertoires to Immunodominant Viral Antigens. Cell Rep. 2017, 19, 569-583. [CrossRef]

23. Emerson, R.; DeWitt, W.S.; Vignali, M.; Gravley, J.; Hu, J.K.; Osborne, E.J.; Desmarais, C.; Klinger, M.; Carlson, C.S.; A Hansen, J.; et al. Immunosequencing identifies signatures of cytomegalovirus exposure history and HLA-mediated effects on the T cell repertoire. Nat. Genet. 2017, 49, 659-665. [CrossRef]

24. Banas, B.; Steubl, D.; Renders, L.; Chittka, D.; Banas, M.C.; Wekerle, T.; Koch, M.; Witzke, O.; Mühlfeld, A.; Sommerer, C.; et al. Clinical validation of a novel enzyme-linked immunosorbent spot assay-basedin vitrodiagnostic assay to monitor cytomegalovirus-specific cell-mediated immunity in kidney transplant recipients: A multicenter, longitudinal, prospective, observational study. Transpl. Int. 2018, 31, 436-450. [CrossRef]

25. Hutchinson, P.; Jose, M.; Atkins, R.C.; Holdsworth, S.R. Ex vivo lymphocyte proliferative function is severely inhibited in renal transplant patients on mycophenolate mofetil treatment. Transpl. Immunol. 2004, 13, 55-61. [CrossRef] [PubMed] 
26. Rentenaar, R.J.; Van Diepen, F.N.; Meijer, R.T.; Surachno, S.; Wilmink, J.M.; Schellekens, P.T.A.; Pals, S.T.; van Lier, R.; Berge, I.J.T. Immune responsiveness in renal transplant recipients: Mycophenolic acid severely depresses humoral immunity in vivo. Kidney Int. 2002, 62, 319-328. [CrossRef] [PubMed]

27. Mulley, W.R.; Visvanathan, K.; Hurt, A.C.; Brown, F.G.; Polkinghorne, K.R.; Mastorakos, T.; Lewicki, M.C.; Stuart, R.L.; Tan, S.-J.; Chean, R.; et al. Mycophenolate and lower graft function reduce the seroresponse of kidney transplant recipients to pandemic H1N1 vaccination. Kidney Int. 2012, 82, 212-219. [CrossRef] [PubMed]

28. Li, W.; Joshi, M.D.; Singhania, S.; Ramsey, K.H.; Murthy, A.K. Peptide Vaccine: Progress and Challenges. Vaccines 2014, 2, 515-536. [CrossRef] [PubMed]

29. Platten, M.; Bunse, L.; Wick, A.; Bunse, T.; Le Cornet, L.; Harting, I.; Sahm, F.; Sanghvi, K.; Tan, C.L.; Poschke, I.; et al. A vaccine targeting mutant IDH1 in newly diagnosed glioma. Nature 2021, 592, 463-468. [CrossRef]

30. Schmitt, A.; Bechter, C.; Yao, J.; Goetz, M.; Maccari, B.; Schauwecker, P.; Wiesneth, M.; Schmitt, M. Cytomegalovirus vaccination of leukemia and lymphoma patients after allogeneic stem cell transplantation-Validation of a peptide vaccine. J. Immunol. Methods 2009, 343, 140-147. [CrossRef]

31. Shugay, M.; Bagaev, D.V.; Zvyagin, I.V.; Vroomans, R.M.; Crawford, J.C.; Dolton, G.; A Komech, E.; Sycheva, A.L.; E Koneva, A.; Egorov, E.S.; et al. VDJdb: A curated database of T-cell receptor sequences with known antigen specificity. Nucleic Acids Res. 2017, 46, D419-D427. [CrossRef] [PubMed] 